\title{
RELACIÓN ENTRE EL NIVEL DE CONOCIMIENTO Y PRÁCTICA EN EL CUIDADO DE LA ADMINISTRACIÓN DE SULFATO DE MAGNESIO, EN CASOS DE PREECLAMPSIA Y ECLAMPSIA EN ENFERMERAS DE EMERGENCIA Y CUIDADOS INTENSIVOS MATERNOS
}

\begin{abstract}
Ana María Garcilaø Laø ${ }^{1}$
RESUMEN

Objetivo. Determinar la relación nivel de conocimientos y las prácticas del cuidado en la administración de sulfato de magnesio en casos de Preeclampsia y Eclampsia. Materiales y métodos. Estudio analítico y descriptivo. La muestra fue 15 Enfermeras de Cuidados Intensivos (UCIM) y 20 enfermeras de Emergencia. La técnica fue la encuesta y la observación directa. Los instrumentos un cuestionario y una lista de chequeo. El cuestionario fue aplicado en reunión mensual de cada servicio, la lista de chequeo se realizó a través de dos observaciones directas en cada turno. La validez estadística del cuestionario fue sometido a la prueba Kuder Richarson y la lista de chequeo fue sometida a la prueba de ítem-test mediante el coeficiente de correlación de Pearson. La recolección de datos fue Junio y Octubre 2013, el procesamiento fue manual por un sistema de paloteo a una tabla matriz codificada. En los resultados se utilizó gráficos y cuadros estadísticos. La medición de la variable independiente: nivel de conocimientos, se asignó a la respuesta correcta el valor de 1 y a la incorrecta 0 . Se aplicó la escala de Sturgest. La medición de la variable dependiente: Practica de Enfermería, se asignó a la respuesta correcta el valor de $1=\mathrm{SI}$ y NO $=0$. Se aplicó la escala de Sturgest. Se obtuvo la aprobación del Comité Ético de Investigación de la institución. Resultados. En la UCIM el conocimiento se ubica en el nivel alto $(33 \%)$, medio (54\%) y bajo (13\%). En cuanto a las prácticas se tiene un nivel bueno ( $40 \%)$, regular $(60 \%)$ y malo $(0 \%)$. En Emergencia, el $25 \%$ tienen conocimientos alto, $60 \%$ medio y bajo el $15 \%$. En las prácticas se encontró solo dos niveles el $45 \%$ bueno y el $55 \%$ regular. La relación entre el nivel de conocimientos y práctica de las enfermeras se pudo verificar a través de la prueba estadística del Ji Cuadrado, con un nivel de significación de 0.05, con 4 grados de libertad, obteniéndose el valor a 6.949, lo cual permitió evidenciar estadísticamente, existe relación entre el nivel de conocimientos y las practicas. Conclusiones. Se puede concluir que el nivel de conocimientos que predominan en las Enfermeras en su mayoría es medio-alto, indica dominio de conocimientos teóricos. Las prácticas en las Enfermeras en su mayoría son medio-altas, se brinda cuidado integral. Existiendo relación entre el nivel de conocimientos y las prácticas y que el nivel de conocimientos influye significativamente en la práctica de enfermería.
\end{abstract}

Palabras claves. Conocimientos, practica, cuidados, sulfato de magnesio, Enfermera.

\section{RELATIONSHIP BETWEEN THE LEVEL OF KNOWLEDGE AND PRACTICE IN THE CARE OF THE ADMINISTRATION OF MAGNESIUM SULPHATE, IN CASES OF PREECLAMPSIA AND ECLAMPSIA IN EMERGENCY NURSES AND MATERNAL INTENSIVE CARE}

\begin{abstract}
Objective. To determine the relationship level of knowledge and care practices in the administration of magnesium sulfate in cases of pre-eclampsia and eclampsia. Material and methods. Analytical and descriptive study. The sample was 15 Intensive Care Nurses (UCIM) and 20 Emergency nurses. The technique was the survey and direct observation. The instruments a questionnaire and a checklist. The questionnaire was applied in monthly meeting of each service, the checklist was made through two direct observations in each shift. The statistical validity of the questionnaire was subjected to the Kuder Richarson test and the checklist was subjected to the item-test using the Pearson correlation coefficient. The data collection was June and October 2013, the processing was manual by a system of paloteo to an encoded matrix table. In the results, graphs and statistical tables were used. The measurement of the independent variable: level of knowledge, was assigned to the correct answer the value of 1 and the incorrect 0 . The Sturgest scale was applied. The measurement of the dependent variable: Nursing Practice, was assigned to the correct answer the value of $1=\mathrm{SI}$ and $\mathrm{NO}=0$. The Sturgest scale was applied. The approval of the Research Ethics Committee of the institution was obtained. Results. In the UCIM the knowledge is located in the high (33\%), medium (54\%) and low (13\%) levels. As for the practices, there is a good $(40 \%)$, regular $(60 \%)$ and bad ( $0 \%)$ level. In Emergency, $25 \%$ have high knowledge, $60 \%$ average and low $15 \%$. In practice, only two levels were found, $45 \%$ good and $55 \%$ regular. The relationship between the level of knowledge and practice of the nurses could be verified through the statistical test of the Chi Square, with a level of significance of 0.05 , with 4 degrees of freedom, obtaining the value at 6.949 , which allowed to show statistically, there is a relationship between the level of knowledge and practices. Conclusions. It can be concluded that the level of knowledge that predominates in Nurses is mostly medium-high, indicating mastery of theoretical knowledge. The practices in Nurses are mostly medium-high, comprehensive care is provided. There is a relationship between the level of knowledge and practices and that the level of knowledge significantly influences the practice of nursing.
\end{abstract}

Keywords. Knowledge, practice, care, magnesium sulfate, nurse.

\section{INTRODUCCIÓN}

La Organización Mundial de la Salud (OMS), indica que diariamente fallecen alrededor de 800 mujeres por causas prevenibles relacionadas con el embarazo y con el parto. Dentro de estas causas la Preeclampsia se encuentra en el tercer lugar, precedida por las hemorragias graves y las infecciones ${ }^{1}$.

La Preeclampsia, es un síndrome multisistémico de severidad variable, específica del embarazo. Se presenta

Lic.Enf.Especialista en Cuidados Intensivos Adulto. Servicio de Cuidados Intensivos Maternos- Instituto Nacional Materno Perinatal. Lima-Perú

Recibido: 10-10-17 Aprobado: 30-12-17 
después de la semana 20 de la gestación, durante el parto o en las primeras 6 semanas después de éste. Esta enfermedad se caracteriza por presentar una respuesta vascular anormal a la placentación y que se asocia a los siguientes cambios: incremento en la respuesta vascular sistémica, aumento en la agregación plaquetaria, activación del sistema de coagulación y a la disfunción celular endotelial. Esta enfermedad de origen desconocido y multifactorial cuyo tratamiento definitivo es el parto, además de ser causal de repercusiones sobre la madre y el recién nacido, es motivo de hospitalizaciones prolongadas y repercusiones económicas para la familia, los establecimientos de salud y el sistema de salud del país ${ }^{3,7}$.

La Eclampsia, su diagnóstico estará dado por la aparición de convulsiones, coma o amaurosis súbita en pacientes con preeclampsia. De acuerdo con el curso clínico y la severidad del compromiso neurológico ${ }^{3,6}$.

A nivel mundial, la incidencia de Preeclampsia oscila entre $2-10 \%$ de los embarazos, la cual es precursor de la eclampsia y varía en todo el mundo. La Organización Mundial de la Salud (OMS) estima que la incidencia de Preeclampsia es siete veces mayor en los países en desarrollo que en los desarrollados $(2,8 \%$ y $0,4 \%$ de los nacidos vivos respectivamente). La incidencia de eclampsia en los países desarrollados de Norteamérica y Europa es similar y se estima alrededor de 5 a 7 casos por cada 10.000 partos, mientras que en países en desarrollo es variable, oscilando entre 1 caso por cada 100 embarazos a 1 por cada 1.700 embarazos $^{5}$.

La Eclampsia es la manifestación más severa de la enfermedad hipertensiva inducida por el embarazo con una incidencia entre 0,5 y 2 por 1000 partos, siendo en los países en vías de desarrollo mucho más elevada llegando hasta el $15 \%$.

El manejo hospitalario de las pacientes con Preeclampsia y Eclampsia es si la presión arterial sistólica es mayor de $30 \mathrm{mmHg}$, y la presión arterial diastólica es mayor de 15 $\mathrm{mmHg}$ con respecto a la basal tomada en 2 ocasiones al azar y con una diferencia mínima de 4 horas. En caso de mujeres en las que no se conozcan las cifras de presión arterial previas, se considerarán cifras a partir de la presión arterial diastólica mayor o igual a $110 \mathrm{mmHg}$. La proteinuria mayor de $5 \mathrm{gr}$ en orina de 24 horas o una prueba de cinta de 3 cruces $(+++)$, en dos ocasiones tomadas al azar y con una diferencia mínima de 4 horas. El edema puede ser generalizado, puede presentarse oliguria y alteraciones visuales y cerebrales, dolor epigástrico, edema pulmonar y trombocitopenia. Las pacientes con preeclampsia grave siempre corren el riesgo de presentar alguna convulsión.

El sulfato de magnesio es el fármaco ideal en el tratamiento y profilaxis de las crisis en las gestantes y puérperas con Preeclampsia y Eclampsia, dado su relativa inocuidad y amplio margen terapéutico, sus efectos son anticonvulsivantes y vasodilatadores ${ }^{8,26}$.
Sibai $^{27}$ indican que independientemente de su eficacia como anticonvulsivante, el Sulfato de Magnesio tiene varios efectos benéficos para la madre preeclámptica, entre los que cabe mencionar incremento del flujo sanguíneo cerebral, rápida disminución del índice de resistencia sistémica vascular, incremento del flujo sanguíneo uterino, incremento del flujo sanguíneo renal, incremento de la liberación de prostaciclinas por las células endoteliales, disminución de la actividad de la renina plasmática, disminución de los niveles de enzima convertidora de angiotensina, atenuación de la respuesta vascular a sustancias vasopresoras, protección contra daño isquémico y reducción de la agregación plaquetaria.

En el 2013 en el Instituto Nacional Materno Perinatal de un total de 1611 pacientes con Diagnósticos de Egreso con Enfermedad Hipertensiva del Embarazo se tiene el Diagnostico de Preeclampsia 1522 que corresponde el $94,5 \%$, Eclampsia 24 corresponde al 1,5\% y Síndrome HELLP 65 que corresponde el $4 \%{ }^{9}$.

El Instituto Nacional Materno Perinatal (INMP) es el centro de referencia a nivel nacional en el sector público por parte del Ministerio de Salud del manejo de pacientes criticas obstétricas con Trastornos Hipertensivos del Embarazo, por esta razón el personal de enfermería debe estar capacitado en el manejo de la atención de pacientes con preeclampsia y eclampsia así como la terapia endovenosa con sulfato de magnesio que incluy entre otros, los procedimientos de instalación, manejo, vigilancia y complicaciones. El personal de enfermería interviene en el plan médico-terapéutico y es responsable de mantener un nivel de experiencia y conocimientos específicos para proporcionar los cuidados necesarios indicados en el plan terapéutico y en atención a las condiciones particulares de cada paciente sobre todo en los servicios de Emergencia y en la Unidad de Cuidados Intensivos Materno. Se planteó el siguiente problema ¿Cuál es la Relación entre el nivel de conocimientos y prácticas hacia el cuidado durante la administración de sulfato de magnesio, en casos de Preeclampsia y Eclampsia en Enfermeras de Emergencia y Cuidados Intensivos Maternos - Instituto Nacional Materno Perinatal?

\section{MATERIALES Y MÉTODOS}

Estudio cuantitativo, analítico y descriptivo. El estudio se realizó en el Instituto Nacional Materno Perinatal de Lima - Perú. Se tuvo como universo muestra al total de Licenciadas en Enfermería que laboran en los servicios de Emergencia y Cuidados Intensivos Maternos que hicieron un total de 35 Enfermeras. Los criterios de inclusión: licenciadas que laboren en Emergencia y Cuidados Intensivos Maternos y que deseen participar del estudio. La técnica que se utiliø fue la encuesta y la observación directa. Los instrumentos fueron un cuestionario y una lista de chequeo. El objetivo fue identificar los conocimientos que tienen las Enfermeras hacia el cuidado durante la 
administración de sulfato de magnesio, en casos de preeclampsia y eclampsia en Enfermeras de Emergencia y Unidad de Cuidados Intensivos Maternos.

La lista de chequeo se realiǿ a través de dos observaciones directas en cada turno de los servicios de Emergencia y Cuidados Intensivos Maternos. El objetivo fue identificar las prácticas hacia el cuidado durante la administración de sulfato de magnesio, en casos de preeclampsia y eclampsia.

\section{Plan de recolección, procesamiento y presentación de datos}

La recolección de datos se realiǿ durante los meses de junio a octubre del 2013. Posteriormente éstos fueron procesados de forma manual mediante un sistema de paloteo a una tabla matriz codificada. Para la presentación de los resultados se utilizó gráficos y cuadros estadísticos para su análisis e interpretación.

Consideraciones éticas. Para el desarrollo del estudio se tomó en cuenta las consideraciones éticas básicas sobre el consentimiento informado a las enfermeras que laboran en Emergencia y Cuidados Intensivos Maternos y la confidencialidad de los datos obtenidos. Además se solicitó anticipadamente la autorización respectiva de las oficinas de docencia e investigación de la institución para la realiz ción del estudio.

\section{RESULTADOS}

Tabla 1. Datos sociodemográficos de las enfermeras de emergencia

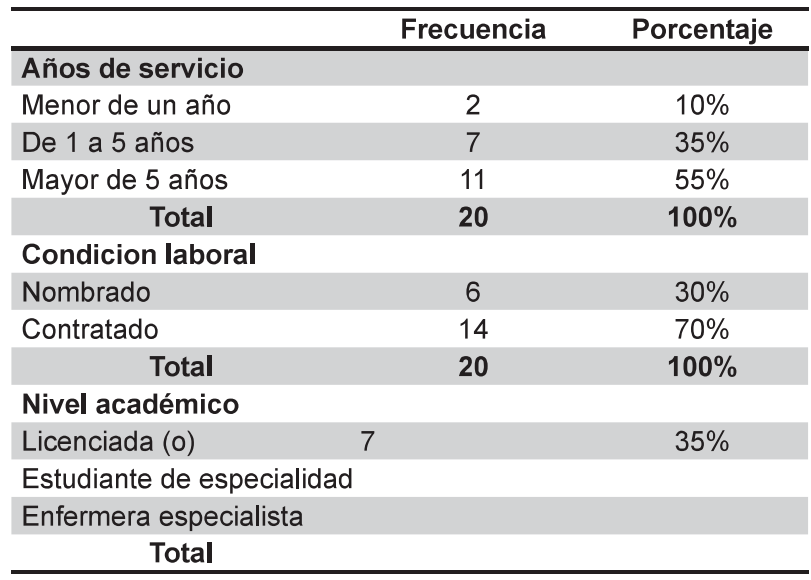

Fuente: Cuestionario aplicado a Enfermeras - Emergencia del INMP 2013

En la tabla 1 se observa que los años de servicio de las enfermeras que laboran en Emergencia fue maø $r$ de 5 años con $55 \%$, de 1 a 5 años $35 \%$ y menor de un año $10 \%$. En su nivel académico el $35 \%$ de las enfermeras de emergencia son licenciadas tituladas, el $50 \%$ son enfermeras tituladas que están estudiando su segunda especialización de Enfermería en Emergencia y el 15\% son enfermeras con doble titulación como licenciadas y como enfermeras especialistas en Emergencia.

Tabla 2. Datos sociodemográficos de las enfermeras de cuidados intensivos maternos

\begin{tabular}{|c|c|c|}
\hline & Frecuencia & Porcentaje \\
\hline \multicolumn{3}{|l|}{ Años de servicio } \\
\hline Menor de un año & 1 & $7 \%$ \\
\hline De 1 a 5 años & 6 & $40 \%$ \\
\hline Mayor de 5 años & 8 & $53 \%$ \\
\hline Total & 15 & $100 \%$ \\
\hline \multicolumn{3}{|l|}{ Condicion laboral } \\
\hline Nombrado & 10 & $67 \%$ \\
\hline Contratado & 5 & $33 \%$ \\
\hline Total & 15 & $100 \%$ \\
\hline \multicolumn{3}{|l|}{ Nivel academico } \\
\hline Licenciada (o) & 0 & $0 \%$ \\
\hline Estudiante de especialidad & 4 & $27 \%$ \\
\hline Enfermera especialista & 11 & $73 \%$ \\
\hline Total & 15 & $100 \%$ \\
\hline
\end{tabular}

Fuente: Cuestionario aplicado a Enfermeras - UCIM del INMP 2013

En la tabla 2 se observa que los años de servicio de las enfermeras que laboran en la Unidad de Cuidados Intensivos Maternos fue mayor de 5 años con $53 \%$, de 1 a 5 años $40 \%$ y menor de un año $7 \%$. En su nivel académico el $73 \%$ son enfermeras con doble titulación como licenciadas y como enfermeras especialistas en Cuidados Intensivos adulto. El $27 \%$ son enfermeras tituladas que están estudiando la segunda especialización en Cuidados Intensivos Adulto.

Tabla 3. Nivel de conocimientos en la administración de sulfato de magnesio, en casos de pre eclampsia y eclampsia en enfermeras de cuidados intensivos maternos (UCIM)

\begin{tabular}{ccc}
\hline $\begin{array}{c}\text { Nivel de } \\
\text { conocimiento-UCIM }\end{array}$ & $\mathbf{N}^{\circ}$ & $\%$ \\
\hline Alto & 5 & $33 \%$ \\
Medio & 8 & $54 \%$ \\
Bajo & 2 & $13 \%$ \\
Total & 15 & $100 \%$ \\
\hline
\end{tabular}

Fuente: Cuestionario aplicado a Enfermeras - UCIM del INMP 2013

Los avances en la tecnología han dado lugar a que cada vez un mayor número de procedimientos de atención directa al paciente alcancen un nivel de especialización y complejidad. Algunos de ellos pueden ser de la responsabilidad única del personal médico o en otros casos atribuibles sólo al personal de enfermería como la administración de medicamentos quien interviene en el plan médico terapéutico y es responsable de mantener un nivel de experiencia y conocimientos específicos para proporcionar los cuidados necesarios indicados en el plan terapéutico y en atención a las condiciones particulares de cada paciente ${ }^{24}$. 
Estos conocimientos permitirán a enfermería implicarse más en el proceso de administración de medicamentos y no solo en su aplicación, lo que traerá consigo un mejor desempeño de sus funciones en la administración de cuidados a los enfermos. Mientras may $r$ sea el conocimiento de la enfermera sobre los medicamentos que administra mayor será su capacidad para desarrollar dicho proceso.

En la tabla 3, se presenta la distribución de 15 enfermeras de UCIM, según valoración del nivel de conocimientos sobre el cuidado del paciente en tratamiento de Preeclampsia y Eclampsia con infusión con sulfato de magnesio se observa que el $54 \%$ de las enfermeras de UCIM tienen un nivel de conocimientos medio, seguido de un $33 \%$ con un nivel alto y $13 \%$ un nivel de conocimientos bajo.

En los resultados encontrados en el presente estudio, se tiene que en su mayoría (54\%) y (33\%) las enfermeras que atienden los casos de preeclampsia y eclampsia con infusión de sulfato de magnesio tienen un nivel de conocimiento regular a bueno, lo cual indica dominio de conocimientos teóricos en esta área; la respuesta a ello se debe a que gran parte de ellas son profesionales con estudios de especialidad en Cuidados Intensivos, por lo que poseen conocimientos especializados sobre procedimientos y administración de medicamentos que le permite valorar la situación y actuar de manera certera contribu ndo a brindar cuidados integrales.

Tabla 4. Nivel de prácticas durante la administración de sulfato de magnesio, en casos de pre eclampsia y eclampsia en UCIM

\begin{tabular}{ccc}
\hline $\begin{array}{c}\text { Nivel de prácticas- } \\
\text { UCIM }\end{array}$ & $\mathbf{N}^{\circ}$ & $\%$ \\
\hline Bueno & 6 & $40 \%$ \\
Regular & 9 & $60 \%$ \\
Malo & 0 & $0 \%$ \\
Total & 15 & $100 \%$ \\
\hline
\end{tabular}

Fuente: Guía de observación tipo Likert aplicado a Enfermeras - UCIM del INMP 2013

La práctica, componente esencial de conocimiento en enfermería, brinda la oportunidad de desarrollar un conocimiento práctico (conocimiento en la acción) Este conocimiento que sólo se adquiere en la "acción" es el que permite posteriormente el reconocimiento rápido de situaciones problemáticas y la respuesta característica de una práctica experta. Es por tanto un conocimiento íntimamente ligado a la experiencia y que se centra en la atención de enfermería a la persona ${ }^{24}$.

Una de las intervenciones de enfermería más frecuentes en la práctica clínica diaria es el mantenimiento y cuidado de la terapia intravenosa, una de las más importantes, ya que alrededor del $60 \%$ de pacientes del ámbito hospitalario, son portadores de un catéter venoso para administración de medicamentos endovenosos. (23)
La enfermera de Cuidados Intensivos debe tener los conocimientos y habilidades para el manejo de pacientes con preeclampsia y eclampsia con infusión de sulfato de magnesio valorar, monitorizar y vigilar posibles complicaciones.

En la tabla 4 se puede observar que la mayoría de las enfermeras de Cuidados Intensivos Maternos (UCIM) presenta de regular a buenas prácticas durante la administración del Sulfato de magnesio, es decir son favorables, brindando un cuidado integral relacionado a la formación profesional donde los conocimientos, las destrezas y habilidades en el manejo de los casos de preeclampsia y eclampsia con infusión de sulfato de magnesio se basara en la valoración integral y permanente, preservación de las funciones a través del monitoreo y prevención de complicaciones, para lo cual se ha de seguir un proceso sistemático de trabajo, establecido en los protocolo de cuidados.

Tabla 5. Relación entre el nivel de conocimientos y prácticas del cuidado durante la administración de sulfato de magnesio, en casos de pre eclampsia y eclampsia en enfermeras UCIM

\begin{tabular}{lcccccccc}
\hline & \multicolumn{6}{c}{ Nivel de conocimientos-UCIM } \\
\cline { 2 - 9 } Prácticas & \multicolumn{3}{c}{ Bueno } & \multicolumn{2}{c}{ Regular } & \multicolumn{2}{c}{ Malo } & \multicolumn{2}{c}{ Total } \\
\cline { 2 - 9 } & $\mathbf{N}^{\circ}$ & $\%$ & $\mathbf{N}^{\circ}$ & $\%$ & $\mathbf{N}^{\circ}$ & $\%$ & $\mathbf{N}^{\circ}$ & $\%$ \\
\hline Alto & 1 & $20 \%$ & 5 & $62 \%$ & 0 & $0 \%$ & 6 & $40 \%$ \\
Medio & 4 & $80 \%$ & 3 & $38 \%$ & 2 & $100 \%$ & 9 & $60 \%$ \\
Bajo & 0 & $0 \%$ & 0 & $0 \%$ & 0 & $0 \%$ & 0 & $0 \%$ \\
Total & $\mathbf{5}$ & $\mathbf{1 0 0} \%$ & $\mathbf{8}$ & $\mathbf{1 0 0} \%$ & $\mathbf{2}$ & $\mathbf{1 0 0} \%$ & $\mathbf{1 5}$ & $\mathbf{1 0 0 \%}$ \\
\hline
\end{tabular}

Fuente: Datos obtenidos del programa SPSS 20. Cuestionario y guía de Observación- INMP-2013

La enfermera utiliza un marco conceptual para organizar los conocimientos adquiridos lo cual utilizara para analizar, intervenir, y evaluar las situaciones de emergencia durante la administración del sulfato de magnesio, es decir, el conocimiento le permitirá actuar de manera adecuada ante las necesidades del paciente.

Así mismo, las prácticas tienen una relación específica con la administración del medicamento, sin embargo a veces la práctica evidencia o confirma si tiene relación con los conocimientos

Siendo la práctica y el conocimiento las bases del cuidado integral del paciente crítico, especialmente en este tipo de pacientes, ya que sólo manteniendo un esfuerzo sostenido durante la perfusión del sulfato de magnesio podremos lograr una respuesta adecuada al tratamiento.

En la tabla 5 dada la relación entre las variables conocimientos y prácticas del cuidado sobre la administración de sulfato de magnesio se observa que de $15(100 \%)$ de enfermeras de UCIM 5 (62\%) tienen un nivel de conocimiento regular con alta práctica, $3(38 \%)$ 
presentan un nivel de conocimiento regular con mediana práctica, $1(20 \%)$ tienen un nivel de conocimiento bueno con alta práctica y $4(80 \%)$ presentan un nivel de conocimiento bueno con mediana práctica.

Esto significa que el nivel de conocimiento que tiene la enfermera en UCIM de regular a bueno, sus prácticas también son de regular a buenas siendo favorable, lo que interpreta que la enfermera además de tener los conocimientos analiz conscientemente la función que tiene con respecto a la administración de sulfato de magnesio en casos de preeclampsia y eclampsia.

Por lo expuesto estadísticamente se encontró un Chicuadrado de 6.949 con un valor $p=0.03<0,05$ por lo que se rechaza la hipótesis nula y se acepta la hipótesis alterna, con lo que se concluy que ex ste relación entre el nivel de conocimientos y prácticas de las Enfermeras hacia el cuidado de las pacientes durante la administración de sulfato de magnesio, en casos de preeclampsia y eclampsia",

Es decir, el nivel de conocimientos que tienen las enfermeras sobre el cuidado de los pacientes con preeclampsia, influye significativamente en las prácticas de enfermería hacia los pacientes durante la administración del sulfato de magnesio.

Según Reyes ${ }^{32}$ muestra la relación entre el nivel de conocimiento de la enfermera sobre administración de medicamentos y número de interacciones medicamentosas, donde se observa que del total de enfermeras con un nivel de conocimiento alto, el mayor porcentaje (98\%) no presentaron interacciones medicamentosas; caso contrario ocurrió con el grupo de enfermeras con un nivel de conocimiento bajo, el mayor porcentaje $(59.2 \%)$ presentaron interacciones medicamentosas. Estos resultados indicaron que a medida que las enfermeras tienen un nivel de conocimiento alto, el número de interacciones medicamentosas disminuye.

La difusión de conocimientos para los profesionales de salud en especial para la enfermera en lo que se refiere a la administración de medicamentos e interacciones asociadas constituye uno de los principales instrumentos de prevención y seguridad en la atención de los pacientes hospitalizados.

Tabla 6. Nivel de conocimientos durante la administración de sulfato de magnesio, en casos de pre eclampsia y eclampsia en enfermeras de emergencia

\begin{tabular}{ccc}
\hline $\begin{array}{c}\text { Nivel de conocimiento- } \\
\text { emergencia }\end{array}$ & $\mathbf{N}^{\circ}$ & $\%$ \\
\hline Alto & 5 & $25 \%$ \\
Medio & 12 & $60 \%$ \\
Bajo & 3 & $15 \%$ \\
Total & $\mathbf{2 0}$ & $\mathbf{1 0 0 \%}$ \\
\hline
\end{tabular}

Fuente: Cuestionario aplicado a Enfermeras -Emergencia del INMP 2013
En la tabla 6, sobre conocimiento de las enfermeras de Emergencia, se observa que el $25 \%$ de las enfermeras tienen un nivel de conocimientos alto, seguido de un $60 \%$ con un nivel medio y en su nivel bajo $15 \%$, lo cual indica dominio de conocimientos teóricos en esta área; la respuesta a ello se debe a que gran parte de ellas son profesionales con estudios de especialidad en Emergencias Obstétricas, por lo que poseen conocimientos especializados sobre procedimientos y administración de medicamentos que le permite valorar la situación y actuar de manera certera contribu ndo a brindar cuidados integrales.

Tabla 7. Nivel de prácticas durante la administración de sulfato de magnesio, en casos de pre eclampsia y eclampsia en enfermeras de emergencia

\begin{tabular}{ccc}
\hline $\begin{array}{c}\text { Nivel de prácticas- } \\
\text { emergencia }\end{array}$ & $\mathbf{N}^{\circ}$ & $\%$ \\
\hline Bueno & 9 & $45 \%$ \\
Regular & 11 & $55 \%$ \\
Malo & 0 & $0 \%$ \\
Total & 20 & $100 \%$ \\
\hline
\end{tabular}

Fuente: Guía de observación tipo Likert aplicado a Enfermeras-Emergencia INMP-2013

En la tabla 7, se encontró solo dos niveles valorados para la práctica de enfermería en Emergencia, el $45 \%$ de las enfermeras tienen un nivel de práctica bueno y el $55 \%$ con un nivel regular, no se encontraron nivel malo durante la práctica de enfermería, es decir las prácticas de Enfermería son favorables, brindando un cuidado integral relacionado a la formación profesional donde los conocimientos, las destrezas y habilidades en el manejo de los casos de preeclampsia y eclampsia con infusión de sulfato de magnesio se basa en la valoración integral y permanente, preservación de las funciones a través del monitoreo y prevención de complicaciones, para lo cual se ha de seguir un proceso sistemático de trabajo, establecido en el protocolo de cuidados.

En la tabla 8 dada la relación entre las variables conocimientos y prácticas del cuidado sobre la administración de sulfato de magnesio se observa que de $20(100 \%)$ de enfermeras de Emergencia, 2(40\%)

Tabla 8. Relación entre el nivel de conocimientos y prácticas del cuidado durante la administración de sulfato de magnesio, en casos de pre eclampsia y eclampsia en enfermeras de emergencia

\begin{tabular}{lcccccccc}
\hline & \multicolumn{4}{c}{ Nivel de conocimientos-emergencia } \\
\cline { 2 - 9 } Practicas & \multicolumn{2}{c}{ Bueno } & \multicolumn{2}{c}{ Regular } & \multicolumn{2}{c}{ Malo } & \multicolumn{2}{c}{ Total } \\
\cline { 2 - 9 } & $\mathbf{N}^{\circ}$ & $\%$ & $\mathbf{N}^{\circ}$ & $\%$ & $\mathbf{N}^{\circ}$ & $\%$ & $\mathbf{N}^{\circ}$ & $\%$ \\
\hline Alto & 2 & $40 \%$ & 7 & $58 \%$ & 0 & $0 \%$ & 9 & $45 \%$ \\
Medio & 3 & $60 \%$ & 5 & $42 \%$ & 3 & $100 \%$ & 11 & $55 \%$ \\
Bajo & 0 & $0 \%$ & 0 & $0 \%$ & 0 & $0 \%$ & 0 & $0 \%$ \\
Total & 5 & $100 \%$ & 12 & $100 \%$ & 3 & $100 \%$ & 20 & $100 \%$ \\
\hline
\end{tabular}

Fuente: Datos obtenidos del programa SPSS 20. Cuestionario y guía de Observación-INMP-2013 
tienen un nivel de conocimiento bueno con alta práctica, $3(60 \%)$ presentan un nivel de conocimiento bueno con mediana práctica, $7(58 \%)$ tienen un nivel de conocimiento regular con alta práctica y $5(75 \%)$ presentan un nivel de conocimiento regular con mediana práctica.

El estudio realiz do por Saucedo et $\mathrm{al}^{30}$ sobre errores frecuentes en la administración de medicamentos intravenosos en pediatría, reportaron que el mayor porcentaje $(68 \%)$ de enfermeras tuvo un nivel de conocimiento eficiente en la administración de medicamentos. Por otro lado, según Quispe ${ }^{31}$ en una evaluación a 14 enfermeras en el Hospital Guillermo Almenara, reportó que tienen un grado de conocimiento regular en cuanto a la administración de fármacos.

Esto significa que el nivel de conocimiento que tiene la enfermera de Emergencia es predominantemente regular y la aplicación de la práctica es medianamente favorable. Según Cisneros ${ }^{29}$ la relación entre el nivel de conocimiento y la aplicación de la práctica de las enfermeras a conocimientos regulares aplican estos menos a sus prácticas y las que tienen buen conocimiento su aplicación en la práctica es mayor.

Las enfermeras de Emergencia poseen los conocimientos, habilidades y destrezas para preparar, administrar y vigilar la administración de sulfato de magnesio pero es importante que se capacite permanentemente, conozca las guías y/o protocolos de manejo y la familiarización con el fármaco.

En esta tabla se puede concluir que la relación entre el nivel de conocimientos y prácticas del cuidado durante la administración de sulfato de magnesio en casos de Preeclampsia y Eclampsia de las Enfermeras en Emergencia influye significativamente el conocimiento en la práctica, por lo que se acepta la hipótesis alterna, para lo cual se utilizó la prueba estadística del Ji Cuadrado, obteniéndose como valor a 6.94 , con un $P$ valor $=0.03<0,05$.

\section{CONCLUSIONES}

- El nivel de conocimientos en la administración de sulfato de magnesio en casos de preeclampsia y eclampsia que predomina en las Enfermeras de Cuidados Intensivos Maternos y Emergencia en su mayoría es medio-alto.

- Las prácticas en la administración de sulfato de magnesio en casos de preeclampsia y eclampsia que predomina en las Enfermeras de Cuidados Intensivos Maternos y Emergencia son en su mayoría medio-alto.

- Existen razones suficientes para afirmar que existe relación significativa entre el nivel de conocimientos y las prácticas del cuidado durante la administración de sulfato de magnesio, en casos de pre eclampsia y eclampsia en enfermeras de Cuidados Intensivos Maternos - Emergencia del Instituto Nacional Materno Perinatal.

Financiamiento: Autofinanciado

Conflicto de interés: La autora declara no tener algún conflicto de interes.

\section{REFERENCIAS BIBLIOGRÁFICAS}

1. OMS. Nota informativa $\mathrm{N}^{\circ} 138$. [Internet]. OMS; 2010 [Acceso 10 de octubre del 2011]. Disponible en: http://www.who.int/ mediacentre/ factsheets/fs348/es/.

2. Myers JE, Baker PN. Hypertensive diseases and eclampsia. Curr Opin Obstet Gynecol 2010;14:119-25

3. Vargas VM, Acosta G. La preeclampsia un problema de salud pública mundial. Rev Chil Obstet Ginecol 2012; 77(6): 471 _ 476.

4. McClure EM, Saleem S, Pasha O, Goldenberg RL. Stillbirth in developing countries: a review of causes, risk factors and prevention strategies. J Matern Fetal Neonatal Med 2011; 22:183-90.

5. World Health Organization. Global Program to Conquer Preeclampsia/Eclampsia. 2012. Osungbade KO, Oladunjoye AO. Public Health Perspectives of Preeclampsia in Developing Countries: Implication for Health System Strengthening. J Pregnancy. 2011; 2011: 481095.Published online 2011 April 4. doi:

6. Witllin A. Prevención y tratamiento de las convulsiones eclámpticas. Clínicas obstétricas y ginecológicas. Hipertensión durante el embarazo. McGraw- Hill Interamericana; 2011.p.459-477.

7. Valarino G, Mora A. Eclampsia. Morbilidad y mortalidad materna y perinatal. Rev Obstet Ginecol Venez. 2010;69(3):152-16.

8. Euser AG, Cipolla M. Magnesium sulfate for the treatment of eclampsia: a brief review. stroke. 2011;40(4)1169-1175.

9. INMP. Análisis de la Situación de los Servicios Hospitalarios del Instituto Nacional Materno Perinatal Instituto Nacional Materno Perinatal. Lima. 2013.

10. Jimenez G. Aplicación de un modelo de Cuidado en el servicio de Ginecobstetricia de la Clínica Universitaria Bolivariana. Av Enferm XXV (1):9-18, 2010

11. Altamirano L. Cuidados del profesional de enfermería dirigidos a las puerperas con eclampsia hospitalizadas en la Unidad Clínica de sala de parto del Hospital Universitario de Caracas, 2012.

12. Castilla $M L$ et al. Conocimiento y Práctica del personal de enfermería sobre síndrome hipertensivo gestacional, en el servicio de ginecoobstetricia, Hospital Asunción Juigalpa, enero - junio 2010. Universidad Politécnica de Nicaragua.

13. González NP, Ballardo MG,et al. Conocimiento de la enfermera en la atención a usuario con preeclampsia y eclampsia. Rev. Enfermería Global. 2011;14.

14. Estiú MC. Se benefician las mujeres con preeclampsia y sus niños con el sulfato de magnesio? El Estudio MAGPIE: Una Investigación Clínica Aleatorizada. The Lancet 2002, 359 1877_90.

15. Euser A, Cipolla M. Magnesium Sulfate for the Treatment of Eclampsia: A Brief Review, Stroke 2009; 40;1169-1175; http:// stroke. ahajournals.org/cgi/content/full/40/4/1169.

16. Muñoz B, Riverola J. Gestión del Conocimiento. España: Biblioteca IESE de Gestión de Empresas, Universidad de Navarra; 2010. 
17. Andreu R, Sieber S. La Gestión Integral del Conocimiento y del Aprendizaje. 5ta ed. México: Iberoamericano; 2011.

18. Davenport T, Prusak L. Working Knowledge: How Organizations Manage What They Know. USA: Harvard Business School Press; 2010.

19. Cárdenas JM. Algunas consideraciones sobre epistemología. Enferm. Cardiol. 2007; 7: (3): 1-4.

20. Montesinos G. El conocimiento y la enfermería. Revista Mex cana de Enfermería Cardiológica 2010;10 (1)

21. Kozier B (2010). Conceptos Ítems en la práctica de Enfermería. 4ta. Edición. New York. Ediciones Interamericanas.

22. Lopez M, Riu M, Forner Bscheid. Cuidados enfermeros.Edit. Masson. España. 2011.

23. Rosas MT. Terapia Intravenosa. Trabajo en equipo para evitar complicaciones por catéteres venosos. Rev Enferm IMSS. 2011;7(2):99-103.

24. Santos B, Guerrero D. Administración de medicamentos. Teoría y práctica. Madrid: Díaz de Santos, 2010.

25. Zabalegui A. Administracion de medicamentos y cálculo y dosis. 2da Edición. Editorial Elseiver. España. 2011

26. Duley L, Gulmezoglu AM, Henderson- Smart DJ. Magnesium sulphate and other anticonvulsants for women with preeclampsia. Cochrane Database Syst Rev. 2013; CD000025.

27. Sibai BM. Diagnosis, prevention, and management of eclampsia. Obstet Gynecol 2010; 105:402-10
28. Instituto Nacional Materno Perinatal. Guias de Practica Clinica y de Procedimientos en Obstetricia y Perinatologia. 1 ed. Lima. 2010. P. 71.

29. Cisneros A. Nivel de conocimientos y aplicación de la técnica de administración de la dopamina por enfermeras. Rev. De Ciencias de la Salud 2:1 2007.

30. Saucedo et al. (2008). Errores frecuentes en la administración de medicamento intravenosos en pediatría [consulta: agosto2010]. Base de datos bibliográficos en Rev Enferm Inst Mex Seguro Soc. También disponible en: http://www.imss.gob.mx/NR/ rdonlyres/4AAE225C -3315-4671-867C-DFC3EB52AFDE/0/ RevEnf1052008.pdf-3.

31. Quispe et al. (2006). Grado de conocimiento de la Enfermera acerca de la administración de fármacos en reanimación cardiopulmonar avanzada [Consulta: setiembre2010]. Base de datos Bibliográficos en el distribuidor del Hospital Guillermo Almenara. También disponible en: http://posgrado. upeu.edu.pe/revista/filev2/50

32. Reyes et al. Nivel de conocimiento de la enfermera sobre administración de medicamento e interacciones medicamentosas potenciales en la Unidad de Cuidados Intensivos del Hospital Regional Docente de Trujillo. UCVScienta S(1), Trujillo- Perú, 2013

Correspondencia: Jr. Santa Rosa 941- Lima 1 Correo electrónico: amgl1806@hotmail.com 